\title{
A LAGRANGIAN APPROACH FOR MODELING ROAD COLLISIONS USING SECOND-ORDER MODELS OF TRAFFIC FLOW*
}

\author{
SALISSOU MOUTARI ${ }^{\dagger}$ AND MICHAEL HERTY ${ }^{\ddagger}$
}

\begin{abstract}
In [M. Herty, A. Klein, S. Moutari, V. Schleper, and G. Steinaur, IMA J. Appl. Math., 78(5), 1087-1108, 2013] and [M. Herty and V. Schleper, ZAMM J. Appl. Math. Mech., 91, 763-776, 2011], a macroscopic approach, derived from fluid-dynamics models, has been introduced to infer traffic conditions prone to road traffic collisions along highways' sections. In these studies, the governing equations are coupled within an Eulerian framework, which assumes fixed interfaces between the models. A coupling in Lagrangian coordinates would enable us to get rid of this (not very realistic) assumption. In this paper, we investigate the well-posedness and the suitability of the coupling of the governing equations within the Lagrangian framework. Further, we illustrate some features of the proposed approach through some numerical simulations.
\end{abstract}

Key words. Road traffic collision, traffic flow, second-order model, Lagrangian formulation.

MSC2000. 35L65.

\section{Introduction}

One of the many challenges brought about by the ever increasing growth of road traffic density is road safety. Road traffic safety is a very active research area, and various studies generally based on statistical models have been dedicated to identifying possible causes of road traffic collisions, in particular in the engineering literature; see e.g. [23, 20, 41, 39, 8]. Albeit these methods enable one to identify "black spots" such as road intersections, they are not capable of capturing traffic conditions prone to road traffic collisions along highways sections, where the most deadly and severe collisions usually occur.

A macroscopic approach, derived from fluid-dynamics models, to infer traffic conditions prone to road traffic collisions along highways' sections has been introduced in $[29,31]$. The governing equations, in the devised approach, are based on a coupling of second-order models describing the dynamics of traffic density and traffic velocity along a road section $[2,5,4,37,16,17,42]$. Unlike classical first-order models of traffic flow $[9,38,36]$, second-order models enjoy an interpretation as follow-theleader microscopic models [1], which enables them to inherit microscopic features at the macroscopic scale. Furthermore, second-order type models can also be derived from high-phase space kinetic models [25].

In $[31,29]$, the models of interest have been coupled within Eulerian coordinates, and this assumes fixed interfaces between the models. The major shortcoming of such formulation is the stationarity of the location of the collision, i.e. the location of the collision is assumed to be known a priori. The aim of the study in this paper is to address this limitation by reformulating the problem in Lagrangian coordinates. Such a formulation enables us not only to get rid of the problem of fixed interfaces as well as the stationarity of the location of the collision, but also to capture in principle the dynamics of the trailing vehicle likely to be the source of a rear end collision.

*Received: December 14, 2013; accepted (in revised form): September 19, 2013. Communicated by Lorenzo Pareschi.

${ }^{\dagger}$ CenSSOR, School of Mathematics and Physics, Queen's University Belfast, University Road, Belfast BT7 1NN, United Kingdom (s.moutari@qub.ac.uk).

${ }^{\ddagger}$ RWTH Aachen University, IGPM, Templergraben 55, D-52065 Aachen, Germany (herty@igpm. rwth-aachen.de). 


\section{LAGRANGIAN MACROSCOPIC MODELLING OF ROAD TRAFFIC COLLISIONS}

The coupling of the models in Lagrangian coordinates is carried out using mathematical techniques analogous to those developed for problems of conservation laws in networks $[13,32,26,21,30,22,27,28,14,15,3,19,18,24]$. The central point, in the discussion of the coupling conditions, is the design of suitable Riemann solvers at the interfaces between the models, including the dynamics of the trailing vehicle likely to be the source of the collision.

After a brief introduction to the second-order models of interest and their Lagrangian formulation, we present some known results on Riemann problems and introduce some coupling conditions consistent with the modeling approach introduced in [31] but now in the moving frame. Some analytical results as well as numerical simulations are presented in the remaining part of the paper.

\section{Preliminary discussion}

In recent works, Herty et al. [31, 29] introduced a macroscopic approach to infer traffic conditions prone to road collisions based on the coupling of the Aw-RascleZhang model (AR model in short) $[2,42]$ and (an approximation to) the pressureless gas dynamics model (PGD in short) $[35,6,7,10]$. In this study we revisit the modeling approach within the Lagrangian framework. Note that the AR model does not allow for concentrations whereas the PGD model may allow for concentrations. This will be the key mechanism to model (and predict) possible road traffic collisions.

Mathematical properties of the AR and PGD models have been extensively discussed in the literature; see e.g. [2, 1, 42] and [35, 6, 7, 10], respectively. Therefore, here we only briefly introduce the properties of the models of interest relevant to our study.

In Eulerian coordinates, the conservative formulation of AR [2] writes

$$
\begin{aligned}
\partial_{t} \rho+\partial_{x}(\rho v) & =0, \\
\partial_{t}(\rho w)+\partial_{x}(\rho w v) & =0, \\
w & =v+p(\rho),
\end{aligned}
$$

where $\rho(x, t)$ and $v(x, t)$ denote, respectively, the average local density and the average local velocity of vehicles, both at the position $x$ and the time $t$. The variable $w$ denotes the "preferred velocity" of drivers, whereas the function $0 \leq p(\rho)<\infty$ is the velocity offset between the current velocity of drivers and their preferred velocity. A prototype candidate for the function $p(\rho)$ (see [4]) is

$$
p(\rho)=\left(\frac{1}{\rho}-\frac{1}{\rho_{\max }}\right)^{\gamma}
$$

with $\rho_{\max }$ the maximal density, i.e. when cars are parked bumper to bumper.

In variables $(\rho, v)$ the AR model (2.1)-(2.3) writes:

$$
\partial_{t} U+A(U) \partial_{x} U=0, U=\left(\begin{array}{l}
\rho \\
v
\end{array}\right), A(U)=\left(\begin{array}{cc}
v & \rho \\
0 & v-\rho p^{\prime}(\rho)
\end{array}\right) .
$$

The eigenvalues of the Jacobian matrix $A(U)$ are $\lambda_{1}=v-\rho p^{\prime}(\rho)$ and $\lambda_{2}=v$ and the respective corresponding right eigenvectors are

$$
r_{1}=\left(\begin{array}{c}
1 \\
-p^{\prime}(\rho)
\end{array}\right) \quad \text { and } \quad r_{2}=\left(\begin{array}{l}
1 \\
0
\end{array}\right)
$$


Therefore, the system (2.1)-(2.3) is strictly hyperbolic away from the vacuum (i.e. for $\rho>0)$. Furthermore, the first eigenvalue of the Jacobian matrix $A(U)$ is genuinely nonlinear whereas the second one is linearly degenerate.

Let us consider the following initial data:

$$
(\rho, v)(x, 0)=\left\{\begin{array}{l}
\left(\rho_{l}, v_{l}\right), \text { if } x<0 \\
\left(\rho_{r}, v_{r}\right), \text { if } x>0 .
\end{array}\right.
$$

Then a self-similar solution to the Riemann problem associated to the AR model (2.1)(2.3) with initial data (2.7) consists of the juxtaposition of a wave of the first family (1-shock or 1-rarefaction) associated with the eigenvalue $\lambda_{1}$ and a wave of the second family (2-contact discontinuity) associated with the eigenvalue $\lambda_{2}$. Moreover, shock curves and rarefaction curves coincide for the AR model, and the Riemann invariants associated with the eigenvalues $\lambda_{1}$ and $\lambda_{2}$ are $w(U)=v+p(\rho)$ and $z(U)=v$.

Along waves of the first family $w$ is conserved. Hence, any state on the left $U_{l}=\left(\rho_{l}, v_{l}\right)$ can be connected to a state on the right $U_{r}=\left(\rho_{r}, v_{r}\right)$ by a wave of the first family if and only if

$$
v_{r}+p\left(\rho_{r}\right)=v_{l}+p\left(\rho_{l}\right) .
$$

Then,

- if $v_{r}<v_{l}$, the wave of the first family is a 1-shock traveling with the speed $s=\frac{\rho_{r} v_{r}-\rho_{l} v_{l}}{\rho_{r}-\rho_{l}}$;

- if $v_{r}>v_{l}$, the wave of the first family is a 1-rarefaction wave.

Any state on the left $U_{l}=\left(\rho_{l}, v_{l}\right)$ can be connected to a state on the right $U_{r}=$ $\left(\rho_{r}, v_{r}\right)$ by a wave of the second family if and only if $v_{l}=v_{r}$, in which case the wave of the second family is a 2-contact discontinuity traveling with speed $s=v_{l}=v_{r}$.

For more details about the solution to the Aw-Rascle model, including regularity requirements, we refer the reader to $[2,1]$.

The pressureless gas dynamics (PGD) model e.g. [35, 6, 7, 10], is defined by the following equations:

$$
\begin{aligned}
& \partial_{t} \rho+\partial_{x}(\rho v)=0, \\
& \partial_{t}(\rho v)+\partial_{x}\left(\rho v^{2}\right)=0,
\end{aligned}
$$

where $\rho(x, t)(\geq 0)$ and $v(x, t)(\geq 0)$ denote respectively the local density and the velocity, both at the position $x$ and the time $t$. In [29] a coupling of the AR model and the PGD model has been discussed, and in [31] the coupling of the AR model and an approximation to the PGD model has been investigated. We follow the latter approach in our analysis in the Lagrangian setting below.

\section{Macroscopic modeling of road traffic collisions in Lagrangian coor- dinates}

Among the various reasons leading to road traffic collisions, here we are particularly interested in collisions related to drivers who do not properly anticipate the traffic situation ahead. Our main objective, in this study, is to infer traffic conditions prone to such high-risk situations using macroscopic variables. To achieve this goal, we consider the following setting: on a road section, the collective behaviour of some drivers may change; the traffic dynamics of those drivers who are fully aware and are acting responsibly, i.e. "careful drivers", is governed by the AR model whereas 
the dynamics of those drivers who are unaware of the traffic conditions ahead and are acting carelessly, i.e. "careless drivers", is described using an approximation of the PGD model. In this section, we will justify the motivations of such approach and discuss the well-posedness of the coupling of the AR model with an approximation of the PGD model in Lagrangian coordinates to infer high-risk traffic conditions on highways' sections. For this aim, let us consider the following rescaled formulation of the AR model (2.1)-(2.3):

$$
\begin{aligned}
\partial_{t} \rho^{\varepsilon}+\partial_{x}\left(\rho^{\varepsilon} v^{\varepsilon}\right) & =0, \\
\partial_{t}\left(\rho^{\varepsilon} w^{\varepsilon}\right)+\partial_{x}\left(\rho^{\varepsilon} w^{\varepsilon} v^{\varepsilon}\right) & =0, \\
w^{\varepsilon} & =v^{\varepsilon}+\varepsilon p\left(\rho^{\varepsilon}\right), \varepsilon>0 .
\end{aligned}
$$

Formally, the limit of the rescaled AR model (3.1)-(3.3) when $\varepsilon \longrightarrow 0$ yields the PGD model (2.9)-(2.10). Rigorous results on existence of solutions to the Riemann problem associated with the PGD model exist and they have been investigated extensively; see e.g. $[35,7,33,10,6,11,12,31]$.

Let $\tau^{\varepsilon}=1 / \rho^{\varepsilon}$ denote the specific volume. Then $\tau_{\min }^{\varepsilon}=1 / \rho_{\max }^{\varepsilon}$. Moreover, when the density is normalized then we have $\tau_{\min }^{\varepsilon}=\rho_{\max }^{\varepsilon}=1$. Because $\rho_{\max }$ corresponds to the density where cars are parked bumper to bumper, then $\tau_{\min }^{\varepsilon}$ could be viewed here as the minimal space that could be occupied by a car. Using the specific volume $\tau$, for $\rho>0$, we change the Eulerian coordinates $(x, t)$ into Lagrangian "mass" coordinates $(X, T)$ with

$$
\partial_{x} X=\rho^{\varepsilon}, \partial_{t} X=-\rho^{\varepsilon} v^{\varepsilon}, T=t
$$

or equivalently

$$
\partial_{X} x=1 / \rho^{\varepsilon}=\tau^{\varepsilon}, \partial_{X} t=0, \partial_{T} x=v^{\varepsilon}, \partial_{T} t=1 .
$$

Note that the independent variables in this transformation are $X$ and $T$. The transformation $(x, t) \rightarrow(X, T)$ is well-defined except for vacuum states where $\tau^{\epsilon}$ contains a $\delta$-function. As in gas dynamics [40] and as discussed in [1] solutions to the AR-model in Eulerian and Lagrangian coordinates are equivalent. This equivalence holds not only for strong solutions but also for weak $\left(L^{\infty}\right)$ solutions.

From the previous equations we obtain, within this framework, $X=X(x, t)=$ $\int^{x} \rho^{\varepsilon}(y, t) d y$. Hence, the variable $X(x, t)$ denotes the total mass of vehicles up to point $x$ at time $t$. If $X\left(x, t_{0}\right)=X(x, t), \forall t>t_{0}$, then, from the microscopic point of view, this means that the vehicle initially at position $x_{0}=x\left(t_{0}\right)$ cannot be overtaken $\forall t>t_{0}$. When changing to Lagrangian mass coordinates there is no transformation in time, i.e., $T=t$.

Using the above framework, the rescaled $\mathrm{AR}\left(\mathrm{AR}^{\varepsilon}\right)$ model (3.1)-(3.3) writes

$$
\begin{aligned}
\partial_{T} \tau^{\varepsilon}-\partial_{X}\left(w^{\varepsilon}-\varepsilon p\left(1 / \tau^{\varepsilon}\right)\right) & =0 \\
\partial_{T} w^{\varepsilon} & =0
\end{aligned}
$$

Let us rewrite the system (3.6)-(3.7) in the following form:

$$
\partial_{T} U^{\varepsilon}+A\left(U^{\varepsilon}\right) \partial_{X} U^{\varepsilon}=0
$$

where $U^{\varepsilon}=\left(\begin{array}{c}\tau^{\varepsilon} \\ w^{\varepsilon}\end{array}\right)$ and $A\left(U^{\varepsilon}\right)=\left(\begin{array}{cc}\varepsilon \frac{p^{\prime}\left(1 / \tau^{\varepsilon}\right)}{\left(\tau^{\varepsilon}\right)^{2}} & -1 \\ 0 & 0\end{array}\right)$. 
Then, the eigenvalues of the Jacobian matrix $A\left(U^{\varepsilon}\right)$ are

$$
\lambda_{1}^{\varepsilon}=-\frac{\varepsilon p^{\prime}\left(1 / \tau^{\varepsilon}\right)}{\left(\tau^{\varepsilon}\right)^{2}} \text { and } \lambda_{2}^{\varepsilon}=0,
$$

and their corresponding right eigenvectors are, respectively,

$$
r_{1}^{\varepsilon}=\left(\begin{array}{l}
1 \\
0
\end{array}\right) \text { and } r_{2}^{\varepsilon}=\left(\begin{array}{c}
1 \\
-\varepsilon \frac{p^{\prime}\left(1 / \tau^{\varepsilon}\right)}{\left(\tau^{\varepsilon}\right)^{2}}
\end{array}\right) .
$$

Therefore, for $\varepsilon>0$ and $\rho^{\varepsilon}=\frac{1}{\tau^{\varepsilon}} \neq 0$ the system (3.6)-(3.7) is strictly hyperbolic. Furthermore, because $\nabla \lambda_{1}\left(U^{\varepsilon}\right) \cdot r_{1} \neq 0, \forall U^{\varepsilon}$ and $\nabla \lambda_{2}\left(U^{\varepsilon}\right) \cdot r_{2}=0, \forall U^{\varepsilon}$, for $\varepsilon>0$ and $\rho^{\varepsilon}=\frac{1}{\tau^{\varepsilon}} \neq 0$, the first eigenvalue $\lambda_{1}^{\varepsilon}$ is genuinely nonlinear whereas the second one, $\lambda_{2}^{\varepsilon}$, is linearly degenerate. Hence, the waves associated with $\lambda_{1}^{\varepsilon}$ are rarefactions or shocks, while those associated with $\lambda_{2}^{\varepsilon}$ are contact discontinuities. The $i^{\text {th }}$ Riemann invariant, in the sense of Lax [34], associated with the eigenvalues $\lambda_{1}^{\varepsilon}$ and $\lambda_{2}^{\varepsilon}$ is given by the function $\phi_{i}\left(U^{\varepsilon}\right), i=1,2$ such that

$$
\nabla \phi_{i}\left(U^{\varepsilon}\right) \cdot r_{i}^{\varepsilon}\left(U^{\varepsilon}\right)=0
$$

Hence,

$$
\phi_{1}\left(U^{\varepsilon}\right)=w^{\varepsilon} \text { and } \phi_{2}\left(U^{\varepsilon}\right)=w^{\varepsilon}-\varepsilon p\left(1 / \tau^{\varepsilon}\right) .
$$

3.1. The Riemann problem associated with the system (3.6)-(3.7). The Riemann problem associated with the $\mathrm{AR}^{\varepsilon}$ system (3.6)-(3.7) in Lagrangian coordinates is given by

$$
\left\{\begin{array}{l}
\partial_{T}\left(\begin{array}{c}
\tau^{\varepsilon} \\
w^{\varepsilon}
\end{array}\right)-\partial_{X}\left(\begin{array}{l}
w^{\varepsilon}-\varepsilon p\left(1 / \tau^{\varepsilon}\right) \\
0
\end{array}\right)=\left(\begin{array}{l}
0 \\
0
\end{array}\right), \\
\left(\tau^{\varepsilon}, w^{\varepsilon}\right)(x, 0)=\left\{\begin{array}{l}
\left(\tau_{l}^{\varepsilon}, w_{l}^{\varepsilon}\right), \text { for } x \leq 0, \\
\left(\tau_{r}^{\varepsilon}, w_{r}^{\varepsilon}\right), \text { for } x>0 .
\end{array}\right.
\end{array}\right.
$$

Definition 3.1. Assume we are given the Riemann problem (3.10) and constants $\left(\tau_{k}^{\varepsilon}, w_{k}^{\varepsilon}\right) \in \mathbb{R}^{2}$ and $k \in\{\ell, r\}$. A simple wave is a self-similar, weak solution to (3.6)(3.7) consisting of either a single wave of the first family or a single wave of the second family.

The solution to the Riemann problem (3.10) is a self-similar, weak solution to equations (3.6)-(3.7).

The simple (or elementary) waves associated with (3.10) are characterized as follows. We assume, in the following, that all initial data does not contain the vacuum state $\rho=0$.

1-Rarefaction waves: Let $U_{l}^{\varepsilon}(x, t)=\left(\tau_{l}^{\varepsilon}, w_{l}^{\varepsilon}\right)$ be a given state. Then, the set of states $U_{r}^{\varepsilon}(x, t)=\left(\tau_{r}^{\varepsilon}, w_{r}^{\varepsilon}\right)$, which can be connected to $U_{l}^{\varepsilon}$ through a 1-rarefaction wave, is constructed as follows. Let $U^{\varepsilon}(\xi)$ be a parametrization (for $\xi=\frac{x}{t} \in \mathbb{R}$ ) of the integral curve of the first family (i.e. associated with $\lambda_{1}^{\varepsilon}$ ). Then we have

$$
\frac{d U^{\varepsilon}(\xi)}{d \xi}=\frac{1}{\nabla \lambda_{1}^{\varepsilon}\left(U^{\varepsilon}(\xi)\right) r_{1}^{\varepsilon}\left(U^{\varepsilon}(\xi)\right)} r_{1}^{\varepsilon}\left(U^{\varepsilon}(\xi)\right), \quad \xi_{l} \leq \xi \leq \xi_{r},
$$

with initial data $U^{\varepsilon}\left(\xi_{l}\right)=U_{l}^{\varepsilon}$, and where $\xi_{l}=\lambda_{1}^{\varepsilon}\left(U_{l}^{\varepsilon}\right)$ and $\xi_{r}=\lambda_{1}^{\varepsilon}\left(U_{r}^{\varepsilon}\right)$. 


\section{LAGRANGIAN MACROSCOPIC MODELLING OF ROAD TRAFFIC COLLISIONS}

Therefore,

$$
\left\{\begin{aligned}
\tau^{\varepsilon}(\xi)^{\prime} & =\frac{\left(\tau^{\varepsilon}\right)^{4}}{\varepsilon p^{\prime \prime}\left(1 / \tau^{\varepsilon}\right)+2 \varepsilon \tau^{\varepsilon} p^{\prime}\left(1 / \tau^{\varepsilon}\right)}, & \tau^{\varepsilon}\left(\xi_{l}\right) & =\tau_{l}^{\varepsilon}, \\
w^{\varepsilon}(\xi)^{\prime} & =0, & w^{\varepsilon}\left(\xi_{l}\right) & =w_{l}^{\varepsilon} .
\end{aligned}\right.
$$

Because $w^{\varepsilon}(\xi)=w_{l}^{\varepsilon}, w^{\varepsilon}$ is constant along waves of the first family. Hence, any given state $U_{l}^{\varepsilon}$, on the left, can be connected to any other state on the right, $U_{r}^{\varepsilon}$, through a 1-rarefaction wave if and only if

$$
w^{\varepsilon}\left(U_{l}^{\varepsilon}\right)=w^{\varepsilon}\left(U_{r}^{\varepsilon}\right) \text { and } \lambda_{1}\left(U_{l}^{\varepsilon}\right)<\lambda_{1}^{\varepsilon}\left(U_{r}^{\varepsilon}\right)
$$

1-Shock waves: Let $U_{l}^{\varepsilon}=\left(\tau_{l}^{\varepsilon}, w_{l}^{\varepsilon}\right)$ be a given state. Then, the set of states $U_{r}^{\varepsilon}=\left(\tau_{r}^{\varepsilon}, w_{r}^{\varepsilon}\right)$, to be determined, which can be connected to $U_{l}^{\varepsilon}$ through a discontinuity traveling with speed $s^{\varepsilon}$ needs to satisfy the Rankine-Hugoniot condition, i.e.

$$
s^{\varepsilon}\left[\begin{array}{c}
\tau_{r}^{\varepsilon}-\tau_{l}^{\varepsilon} \\
w_{r}^{\varepsilon}-w_{l}^{\varepsilon}
\end{array}\right]=\left[\begin{array}{c}
\varepsilon p\left(1 / \tau_{r}^{\varepsilon}\right)-w_{r}^{\varepsilon}-\varepsilon p\left(1 / \tau_{l}^{\varepsilon}\right)+w_{l}^{\varepsilon} \\
0
\end{array}\right] .
$$

The case $s^{\varepsilon}=0$ corresponds to a contact discontinuity and this will be discussed in the next section.

For $s^{\varepsilon} \neq 0$, we have

$$
w^{\varepsilon}\left(U_{r}^{\varepsilon}\right)=w^{\varepsilon}\left(U_{l}^{\varepsilon}\right) \text { and } s^{\varepsilon}=\varepsilon \frac{p\left(1 / \tau_{r}^{\varepsilon}\right)-p\left(1 / \tau_{l}^{\varepsilon}\right)}{\tau_{r}^{\varepsilon}-\tau_{l}^{\varepsilon}} .
$$

Using the parametrization $\tau_{r}^{\varepsilon}=\tau_{l}^{\varepsilon}+\xi$, we have

$$
U_{r}^{\varepsilon}\left(\xi, U_{l}^{\varepsilon}\right)=U_{l}^{\varepsilon}+\xi\left(\begin{array}{l}
1 \\
0
\end{array}\right) \text { and } s^{\varepsilon}\left(\xi, U_{l}^{\varepsilon}\right)=\varepsilon \frac{p\left(1 /\left(\tau_{l}^{\varepsilon}+\xi\right)\right)-p\left(1 / \tau_{l}^{\varepsilon}\right)}{\left(\tau_{l}^{\varepsilon}+\xi\right)-\tau_{l}^{\varepsilon}}
$$

Furthermore, it is easy to check that

$$
U_{r}^{\varepsilon}\left(0, U_{l}^{\varepsilon}\right)=U_{l}^{\varepsilon} \text { and } s^{\varepsilon}\left(0, U_{l}^{\varepsilon}\right)=-\varepsilon \frac{p^{\prime}\left(1 / \tau_{l}^{\varepsilon}\right)}{\left(\tau_{l}^{\varepsilon}\right)^{2}}=\lambda_{1}^{\varepsilon}\left(U_{l}^{\varepsilon}\right)
$$

For waves of the first family, shock and rarefaction curves coincide, thus the system (3.6)-(3.7) forms a Temple system. Any given state $U_{l}^{\varepsilon}$, on the left, can be connected to any other state on the right $U_{r}^{\varepsilon}$ through a 1-shock wave of speed $s^{\varepsilon}$ if and only if

$$
w^{\varepsilon}\left(U_{r}^{\varepsilon}\right)=w^{\varepsilon}\left(U_{l}^{\varepsilon}\right) \text { and }-\varepsilon \frac{p^{\prime}\left(1 / \tau_{r}^{\varepsilon}\right)}{\left(\tau_{r}^{\varepsilon}\right)^{2}}=\lambda_{1}^{\varepsilon}\left(U_{r}^{\varepsilon}\right) \leq s^{\varepsilon} \leq \lambda_{1}^{\varepsilon}\left(U_{l}^{\varepsilon}\right)=-\varepsilon \frac{p^{\prime}\left(1 / \tau_{l}^{\varepsilon}\right)}{\left(\tau_{l}^{\varepsilon}\right)^{2}} \text {. }
$$

2-Contact discontinuities: This case consists of solutions to equation (3.13) with $s^{\varepsilon}=0$. Hence, any given state $U_{l}^{\varepsilon}$, on the left, can be connected to any other state on the right, $U_{r}^{\varepsilon}$, through a 2-contact discontinuity (with speed $s^{\varepsilon}=\lambda_{2}^{\varepsilon}\left(U^{\varepsilon}\right)=0$ ) if and only if

$$
w_{r}^{\varepsilon}=w_{l}^{\varepsilon}-\varepsilon p\left(1 / \tau_{l}^{\varepsilon}\right)+\varepsilon p\left(1 / \tau_{r}^{\varepsilon}\right)
$$

i.e. $w_{r}^{\varepsilon}=\varepsilon p\left(1 / \tau_{r}^{\varepsilon}\right)+$ constant .

Following the above discussion on the structure of the waves associated to the Riemann problem (3.10), we have the following results. 


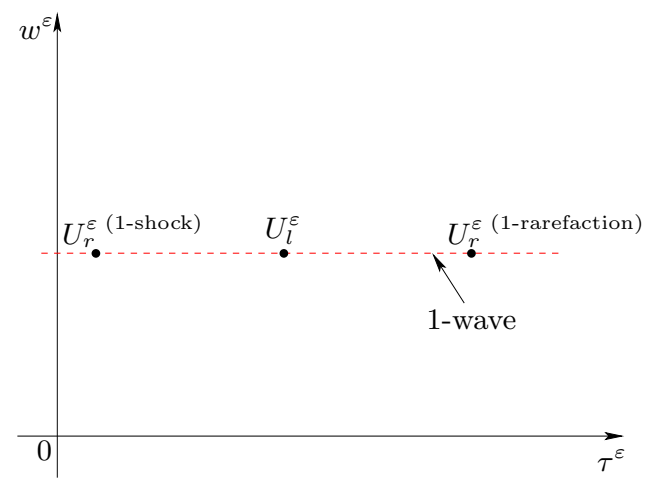

FIG. 3.1. The 1-shock and 1-rarefaction curves for the $A R^{\varepsilon}$ model in the Lagrangian phase plane $\left(\tau^{\varepsilon}, w^{\varepsilon}\right)$.

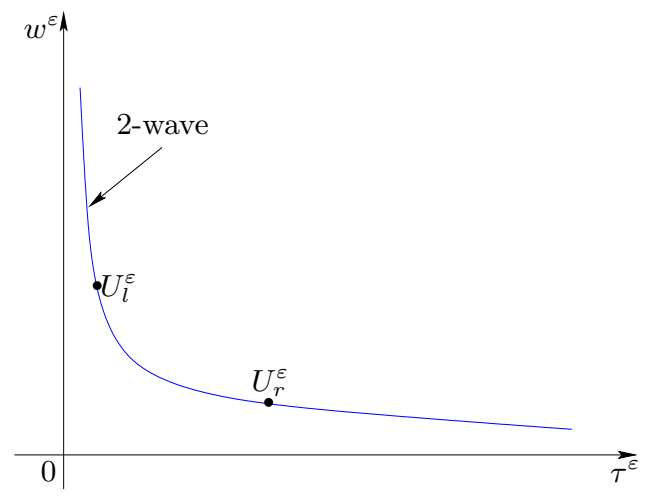

FIG. 3.2. The 2-contact discontinuity curve for the rescaled $A R^{\varepsilon}$ model in the Lagrangian phase plane $\left(\tau^{\varepsilon}, w^{\varepsilon}\right)$.

Waves of the first family: We obtain the wave of the first family when a state, on the left, $U_{l}^{\varepsilon}=\left(\tau_{l}^{\varepsilon}, w_{l}^{\varepsilon}\right)$ is connected with a state, on the right, $U_{r}^{\varepsilon}=\left(\tau_{r}^{\varepsilon}, w_{r}^{\varepsilon}\right)$ through the curve

$$
w_{r}^{\varepsilon}=w_{l}^{\varepsilon}
$$

We can distinguish two cases:

1. If $p\left(1 / \tau_{r}^{\varepsilon}\right)>p\left(1 / \tau_{l}^{\varepsilon}\right)$, then the wave of the first family is a 1-shock, i.e. a jump discontinuity, traveling with the speed

$$
s^{\varepsilon}=\varepsilon \frac{p\left(1 / \tau_{r}^{\varepsilon}\right)-p\left(1 / \tau_{l}^{\varepsilon}\right)}{\tau_{r}^{\varepsilon}-\tau_{l}^{\varepsilon}} .
$$

2. On the other hand, if $p\left(1 / \tau_{r}^{\varepsilon}\right)<p\left(1 / \tau_{l}^{\varepsilon}\right)$, then the wave of the first family is a 1-rarefaction i.e. a continuous solution of the form $U^{\varepsilon}(\xi)=\left(\tau^{\varepsilon}, w^{\varepsilon}\right)(\xi)$ (with 
$\left.\xi=\frac{x}{t}\right)$ given by

$$
\begin{array}{r}
\left(\begin{array}{c}
\left(\tau^{\varepsilon}\right)^{\prime}(\xi) \\
\left(w^{\varepsilon}\right)^{\prime}(\xi)
\end{array}\right)=\frac{r_{1}\left(U^{\varepsilon}(\xi)\right)}{\nabla \lambda_{1}^{\varepsilon}\left(U^{\varepsilon}(\xi)\right) \cdot r_{1}^{\varepsilon}\left(U^{\varepsilon}(\xi)\right)}, \lambda_{1}^{\varepsilon}\left(U_{l}^{\varepsilon}\right) \leq \xi \leq \lambda_{1}^{\varepsilon}\left(U_{r}^{\varepsilon}\right), \\
\left(\tau^{\varepsilon}, w^{\varepsilon}\right)(\xi)=\left\{\begin{array}{lll}
\left(\tau_{l}^{\varepsilon}, w_{l}^{\varepsilon}\right), & \text { for } & \xi<\lambda_{1}^{\varepsilon}\left(U_{l}^{\varepsilon}\right), \\
\left(\tau_{r}^{\varepsilon}, w_{r}^{\varepsilon}\right), & \text { for } & \xi>\lambda_{2}^{\varepsilon}\left(U_{r}^{\varepsilon}\right) .
\end{array}\right.
\end{array}
$$

Waves of the second family: We obtain a wave of the second family, i.e. a 2 -contact discontinuity, when

$$
w_{r}^{\varepsilon}=w_{l}^{\varepsilon}-\varepsilon p\left(1 / \tau_{l}^{\varepsilon}\right)+\varepsilon p\left(1 / \tau_{r}^{\varepsilon}\right),
$$

Proposition 3.2. Let $U_{l}^{\varepsilon}=\left(\tau_{l}^{\varepsilon}, w_{l}^{\varepsilon}\right)$ and $U_{r}^{\varepsilon}=\left(\tau_{r}^{\varepsilon}, w_{r}^{\varepsilon}\right)$ be two given states with $\tau_{l, r}^{\varepsilon} \neq 0$. Provided that either $w_{l}^{\varepsilon}>w_{r}^{\varepsilon}-\varepsilon p\left(1 / \tau_{r}^{\varepsilon}\right)$ or $w_{l}^{\varepsilon}-\varepsilon p\left(1 / \tau_{l}^{\varepsilon}\right)<w_{r}^{\varepsilon}-\varepsilon p\left(1 / \tau_{r}^{\varepsilon}\right)<$ $w_{l}^{\varepsilon}$ holds, a solution to the Riemann problem exists. The solution to the Riemann problem (3.10) is a juxtaposition of two simple waves separated by an intermediate state $\bar{U}^{\varepsilon}=\left(\bar{\tau}^{\varepsilon}, \bar{w}^{\varepsilon}\right)$.

Note that the intermediate state is the intersection point between the 1-wave curve through $U_{l}^{\varepsilon}$ and the 2-contact discontinuity through $U_{r}^{\varepsilon}$.

Proof. The solution is constructed as follows: First, the state on the left, $U_{l}^{\varepsilon}$, is connected to the intermediate state $\bar{U}^{\varepsilon}$ through either a 1-shock or a 1-rarefaction wave, and then $\bar{U}^{\varepsilon}$ is connected to the state on the right, $U_{r}^{\varepsilon}$, through a 2-contact discontinuity. Thus, we have

$$
\bar{w}^{\varepsilon}=w_{l}^{\varepsilon} \quad \text { and } \quad \bar{w}^{\varepsilon}-\varepsilon p\left(1 / \bar{\tau}^{\varepsilon}\right)=w_{r}^{\varepsilon}-\varepsilon p\left(1 / \tau_{r}^{\varepsilon}\right) .
$$

Hence, the intermediate state is given by $\bar{U}^{\varepsilon}=\left(\bar{\tau}^{\varepsilon}, \bar{w}^{\varepsilon}\right)$, where $\bar{w}^{\varepsilon}=w_{l}^{\varepsilon}$ and

$$
\bar{\tau}^{\varepsilon}=\frac{1}{p^{-1}\left(\varepsilon^{-1}\left(w_{l}^{\varepsilon}-w_{r}^{\varepsilon}\right)+p\left(1 / \tau_{r}^{\varepsilon}\right)\right)} .
$$

Because the function $p(\cdot)$ is defined on $\mathbb{R}^{+}$, equation (3.24) admits a finite solution (i.e. non vacuum solution) if and only if $w_{l}^{\varepsilon}>w_{r}^{\varepsilon}-\varepsilon p\left(1 / \tau_{r}^{\varepsilon}\right)$. Therefore, the solution to the Riemann problem (3.10) consists of one of the following cases:

- Case 1: $w_{l}^{\varepsilon} \geq w_{l}^{\varepsilon}-\varepsilon p\left(1 / \tau_{l}^{\varepsilon}\right)>w_{r}^{\varepsilon}-\varepsilon p\left(1 / \tau_{r}^{\varepsilon}\right)$.

In this case, the state $U_{l}^{\varepsilon}$ is connected to the intermediate state $\bar{U}^{\varepsilon}=\left(\bar{\tau}^{\varepsilon}, w_{l}^{\varepsilon}\right)$ (with $\bar{\tau}^{\varepsilon}$ given by (3.24)) through a 1 -shock wave, and then $\bar{U}^{\varepsilon}$ is connected to $U_{r}^{\varepsilon}$ through a 2-contact discontinuity.

- Case 2: $w_{l}^{\varepsilon}-\varepsilon p\left(1 / \tau_{l}^{\varepsilon}\right)<w_{r}^{\varepsilon}-\varepsilon p\left(1 / \tau_{r}^{\varepsilon}\right)<w_{l}^{\varepsilon}$.

In this case, the state $U_{l}^{\varepsilon}$ is connected to the intermediate state $\bar{U}^{\varepsilon}=\left(\bar{\tau}^{\varepsilon}, w_{l}^{\varepsilon}\right)$ (with $\bar{\tau}^{\varepsilon}$ given by (3.24)) through a 1-rarefaction wave, and then $\bar{U}^{\varepsilon}$ is connected to $U_{r}^{\varepsilon}$ through a 2-contact discontinuity.

Concerning the assumption on the initial data we have the following remark. Assume $w_{l}^{\varepsilon}-\varepsilon p\left(1 / \tau_{l}^{\varepsilon}\right)<w_{l}^{\varepsilon}<w_{r}^{\varepsilon}-\varepsilon p\left(1 / \tau_{r}^{\varepsilon}\right)$. In this case, the state $U_{l}^{\varepsilon}$ needs to be connected to the intermediate state $\bar{U}^{\varepsilon}=\left(\infty, w_{l}^{\varepsilon}\right)$ through a 1-rarefaction wave, and then connected to $U_{r}^{\varepsilon}$ through a 2-contact discontinuity. Note that $U^{\varepsilon}$ is not a welldefined state in the Lagrangian coordinate system, because $\tau^{*} \rightarrow \infty$. However, in the Eulerian coordinates this state exists and corresponds to the vacuum $\rho=0$. 


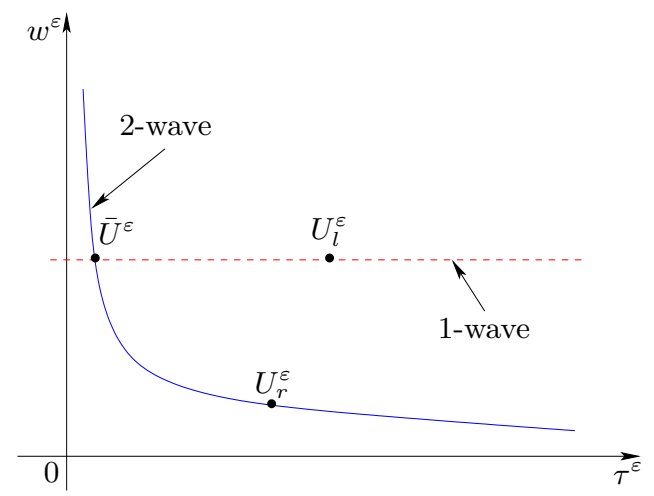

FIG. 3.3. A non vacuum solution to the Riemann problem (3.10) in the Lagrangian phase plane $\left(\tau^{\varepsilon}, w^{\varepsilon}\right)$.

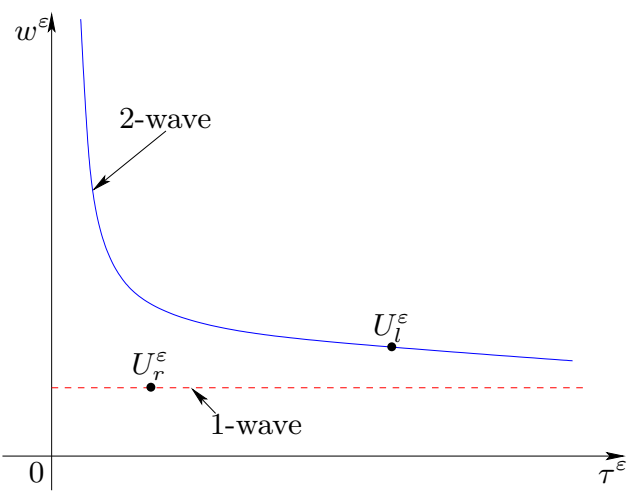

FIG. 3.4. A vacuum solution to the Riemann problem (3.10) in the Lagrangian phase plane $\left(\tau^{\varepsilon}, w^{\varepsilon}\right)$.

3.2. Derivation of a macroscopic model for road traffic collisions in Lagrangian coordinates. As in [31], we now couple the $\mathrm{AR}^{\varepsilon}$ model (3.1)-(3.3) and the AR model (2.1)-(2.3). However, unlike in [31], here we proceed in Lagrangian coordinates, which enables us to track the dynamics of the trailing vehicle likely to be the source of a rear end collision. Instead of simply prescribing coupling conditions as in $[31,29]$, here we start with the following formulation having two traffic pressures on either side of the car likely to be the source of the collision (see figure 3.5 ):

$$
\begin{aligned}
\partial_{t} \rho+\partial_{x}(\rho v) & =0, \\
\partial_{t}(\rho w)+\partial_{x}(\rho w v) & =0, \\
w & =v+\varepsilon p(\rho)+H\left(x-x^{*}\right)(1-\varepsilon) p(\rho), \varepsilon>0,
\end{aligned}
$$

where $H(\cdot)$ denotes the Heaviside function.

The model (3.25)-(3.27) couples the AR model (2.1)-(2.3) and the $\mathrm{AR}^{\varepsilon}$ model (3.1)-(3.3). More precisely, for $x>x^{*}$ the traffic dynamics is governed by the AR model whereas for $x<x^{*}$ the dynamics is driven by the $\mathrm{AR}^{\varepsilon}$ model. The position $x^{*}=x^{*}(t)$ is the trajectory of the car likely to be the source of the collision. Thus, in 
Lagrangian coordinates, the system (3.25)-(3.27) is equivalent to

$$
\begin{aligned}
& \partial_{T} \tau-\partial_{X}(w-p(1 / \tau))=0, \\
& \partial_{T} w=0, \\
& w=v+p(1 / \tau), \\
& \\
& \partial_{T} \tau^{\varepsilon}-\partial_{X}\left(w^{\varepsilon}-\varepsilon p\left(1 / \tau^{\varepsilon}\right)\right)=0, \\
& \partial_{T} w^{\varepsilon}=0, \\
& w^{\varepsilon}=v^{\varepsilon}+\varepsilon p\left(1 / \tau^{\varepsilon}\right),
\end{aligned}
$$

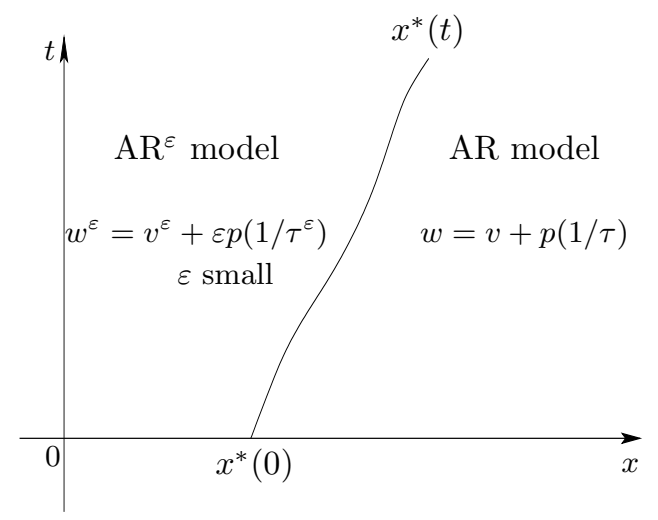

FIG. 3.5. Traffic dynamics of the coupled $A R^{\varepsilon}$ and $A R$ models in Eulerian coordinates.

The coupled model (3.28)-(3.29) assumes that cars cannot overtake each other, and this can be formulated as follows. Given the initial condition and an assigned position $x_{0}^{*}=x^{*}(0)$ (i.e. a car), then the value of $X\left(x^{*}, t\right)=X\left(x_{0}^{*}\right)=\int^{x_{0}^{*}} \rho_{0}(y) d y$ remains unchanged; therefore $X\left(x^{*}, t\right)=$ constant can be viewed as the index of the car which was initially at the location $x_{0}^{*}=x^{*}(0)$. If we assume that we know a priori which car, i.e. $x_{0}$, is likely to be the source of the collision and $\rho_{0}(y)$, then the dynamics of the cars in front of $x_{0}$ is governed by equation (3.28) whereas the dynamics of the cars behind $x_{0}$ is described by equation (3.29). In other words, for any time $t \geq 0$, cars at a position less than or equal to $x^{*}(t)$ are driven carelessly while those at a position greater than $x^{*}(t)$ are driven carefully. Instead of fixing the position $x^{*}(t)$ of the collision (as in [31]), here we only fix the index of the car $X\left(x^{*}(t), t\right)=X\left(x_{0}^{*}\right)$ which is likely to be the source of the collision. The rationale behind the use of Lagrangian coordinates to investigate occurrence of road traffic collision can be summarized as follows: within the Lagrangian framework, the car index remains fixed and this enables us to separate domains where the actual trajectories of the cars likely to be involving in the collision are still varying in time. Note that as in [29] the occurrence of a collision then still depends on the local traffic state.

Next, we investigate whether the Riemann problem associated with (3.25)-(3.27) admits a solution. Throughout the following discussion the quantities $\tau, v$, and $w=$ $v+p(1 / \tau)$ will be associated with the traffic dynamics governed by the AR model 
whereas $\tau^{\varepsilon}$, $v^{\varepsilon}$, and $w^{\varepsilon}=v^{\varepsilon}+\varepsilon p\left(1 / \tau^{\varepsilon}\right)$ will be used to describe the traffic regime governed by the $\mathrm{AR}^{\varepsilon}$ model, as indicated in figure 3.5.

Proposition 3.3. Let $U^{\varepsilon}=\left(\tau^{\varepsilon}, w^{\varepsilon}\right)$ and $U=(\tau, w)$ be two given states in the road sections governed by the $A R^{\varepsilon}$ model and the $A R$ model, respectively, as depicted in figure 3.5. We assume that $\tau^{\varepsilon} \neq 0, \tau \neq 0$, and $w^{\varepsilon}>w-p(1 / \tau)$. Then, the solution to the Riemann problem associated with the model (3.28)-(3.29) is given by a juxtaposition of two simple waves separated by an intermediate state $U^{*}=\left(\tau^{*}, w^{*}\right)$. The intermediate state $U^{*}$ corresponds to the intersection point between a $1^{\varepsilon}$-wave curve emerging from $U^{\varepsilon}$ and the 2-wave curve emerging from $U$.

Proof. The solution is to connect the state, on the left, $U^{\varepsilon}$, to the intermediate state $U^{*}$ through either a $1^{\varepsilon}$-shock or a $1^{\varepsilon}$-rarefaction, and then connect $U^{*}$ to the state on the right, $U$, through a 2-contact discontinuity. Under the assumption $w^{\varepsilon}>$ $w-p(1 / \tau)$ we obtain the following:

1. Case 1: If $w^{\varepsilon}-\varepsilon p\left(1 / \tau^{\varepsilon}\right)<w-p(1 / \tau)<w^{\varepsilon}$, then the solution is to connect the state on the left, $U^{\varepsilon}$, to the intermediate state $U^{*}$ through a $1^{\varepsilon}$-rarefaction wave, and then connect $U^{*}$ to the state on the right, $U$, through a 2-contact discontinuity.

2. Case 2: If $w^{\varepsilon}-\varepsilon p\left(1 / \tau^{\varepsilon}\right)>w-p(1 / \tau)$, then the solution is to connect the state on the left, $U^{\varepsilon}$, to the intermediate state $U^{*}$ through a $1^{\varepsilon}$-shock wave, and then connect $U^{*}$ to the state on the right, $U$, through a 2-contact discontinuity.

Thus, in both sub-cases, we have $U^{*}=\left(\tau^{*}, w^{*}\right)$, where $w^{*}=w^{\varepsilon}$ and

$$
\tau^{*}=\frac{1}{p^{-1}\left(\varepsilon^{-1}\left[\left(w^{\varepsilon}-w\right)+p\left(1 / \tau^{\varepsilon}\right)\right]\right)} .
$$

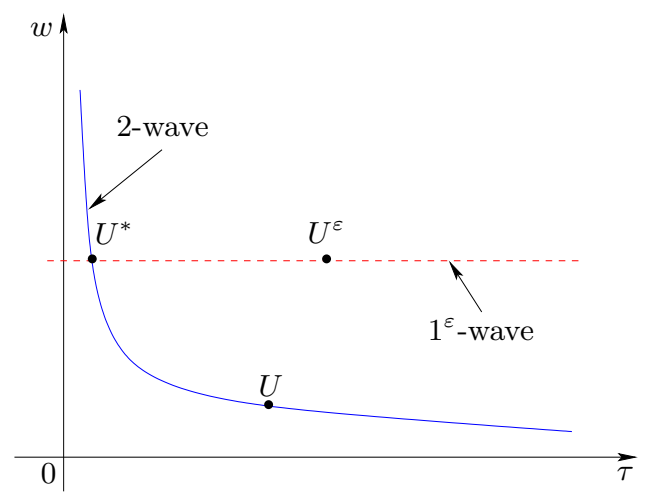

FIG. 3.6. The non-vacuum solution to the Riemann problem associated with the model (3.28)(3.29), in the Lagrangian phase plane $(\tau, w)$.

If the assumption on the initial data is violated, i.e., if $w^{\varepsilon} \leq w-p(1 / \tau)$, then we obtain the following result. A 1-rarefaction wave connects the state $U^{\varepsilon}$ to the state $U^{*}=\left(\infty, w^{\varepsilon}\right)$, and then $U^{*}$ is connected to $U$ through a 2-contact discontinuity. Again, in Eulerian coordinates this corresponds to the vacuum state $\rho^{*}=0$.

In [29] it has been argued that the existence of $\delta$-shocks in the density will be interpreted as the occurrence of a collision at the position $x^{*}(t)$. For $\varepsilon>0$, there are 


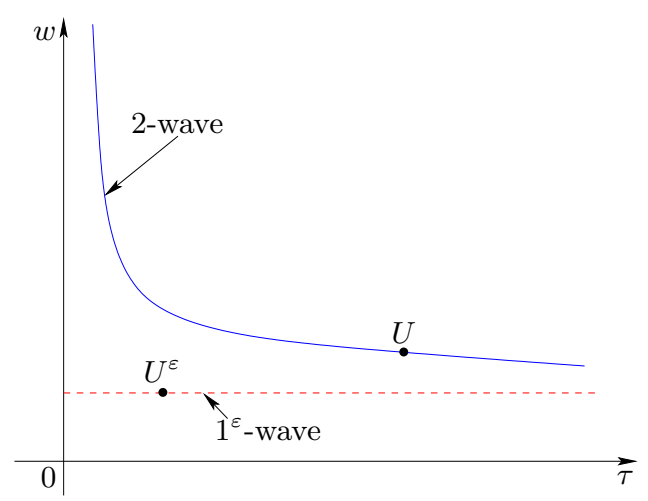

FIG. 3.7. The vacuum solution to the Riemann problem associated with the model (3.28)-(3.29), in the Lagrangian phase plane $(\tau, w)$.

no $\delta$-shock solutions in the model (3.28)-(3.29). However, the variable $\tau=\frac{1}{\rho}$ can be interpreted as the spacing between consecutive cars. Hence, if the spacing tends to zero the car density tends to infinity and this is viewed as an occurrence of a collision in the coupled model (3.28)-(3.29). The mathematical discussion of the situation can be carried out as follows. Let $U^{*}=\left(\tau^{*}, w^{*}\right)$ be the intermediate state of a non-vacuum solution to the Riemann problem associated with the model (3.28)-(3.29), and let $\tau_{\min }$ denote the minimal spatial occupancy of a single car. Then, we have the following:

1. If $\tau^{*}>\tau_{\min }$, the solution consists of a 1-rarefaction wave connecting the state $U^{\varepsilon}$ to an intermediate state $U^{*}=\left(\tau^{*}, w^{\varepsilon}\right)$, followed by a 2-contact discontinuity connecting $U^{*}$ to $U$.

2. If $\tau^{*}<\tau_{\min }$, the solution consists of a 1-shock wave connecting the state $U^{\varepsilon}$ to an intermediate state $U^{*}=\left(\tau^{*}, w^{\varepsilon}\right)$, followed by a 2-contact discontinuity connecting $U^{*}$ to $U$.

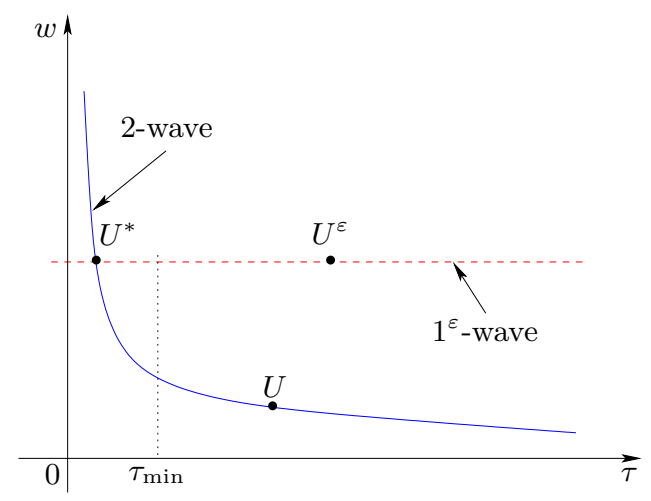

FIG. 3.8. The non-vacuum solution, to the Riemann problem associated with the model (3.28)(3.29), in the case of collision, in the Lagrangian phase plane $(\tau, w)$.

Finally, we turn to special initial data in order to investigate if there is a possibility 
to fulfill $\tau^{*}<\tau_{\min }$. Assume a family of initial data $\left(U^{\epsilon}\right)_{\epsilon}$ such that

$$
\lim _{\varepsilon \longrightarrow 0}\left(\varepsilon^{-1}\left[\left(w^{\varepsilon}-w\right)+p\left(1 / \tau^{\varepsilon}\right)\right]\right) \longrightarrow \infty .
$$

Then, there exists an $\varepsilon$ sufficiently small such that $\tau^{*}<\tau_{\min }$. Because $p(\cdot)$ is a monotone function, we have

$$
\lim _{\varepsilon \longrightarrow 0} p^{-1}\left(\varepsilon^{-1}\left[\left(w^{\varepsilon}-w\right)+p\left(1 / \tau^{\varepsilon}\right)\right]\right) \longrightarrow \infty .
$$

Hence, from (3.30) we have $\tau^{*} \longrightarrow 0$. Therefore, for $\varepsilon$ sufficiently small, there exists $\tau_{\min }>0$ such that $\tau^{*}<\tau_{\min }$. The previous assumption (3.31) is fulfilled for example for all initial data $U^{\varepsilon}$ of the type $\left(\tau^{\varepsilon}, w^{\varepsilon}\right)$ constant and independent of $\varepsilon$.

The above results can be summarized as follows.

Proposition 3.4. Let $\tau_{\min }>0$ denote the minimal spatial occupancy allowed for a single car, and let $U^{\varepsilon}=\left(\tau^{\varepsilon}, w^{\varepsilon}\right)$ and $U=(\tau, w)$ be two given constant states on the road sections governed by the $A R^{\varepsilon}$ model and the $A R$ model, respectively. Assume $w^{\varepsilon}>w-p(1 / \tau)$.

Then, the solution to the Riemann problem associated with the model (3.28)-(3.29) consists of one of the following:

1. If $w^{\varepsilon}=w-p(1 / \tau)$, then the solution is a contact discontinuity with speed $w-p(1 / \tau)$ separating the states $U^{\varepsilon}$ and $U$.

2. If $w^{\varepsilon}>w-p(1 / \tau)$, then the solution is given by a $1^{\varepsilon}$-shock connected to an intermediate state $U^{*}=\left(\tau^{*}, w^{\varepsilon}\right)$ followed by a 2 -contact discontinuity.

If $w^{\varepsilon}<w-p(1 / \tau)$, then the solution consists of two contact discontinuities (with speed $w^{\varepsilon}$ and $w-p(1 / \tau)$, respectively). The intermediate state is only well-defined in Eulerian coordinates and it corresponds to the vacuum in $\rho$, i.e. $\rho=0$.

Note that Case 2 in Proposition 3.4 corresponds to a situation where a collision occurs at $x^{*}(t)$. Therefore, the condition of occurrence of such a collision within the model (3.28)-(3.29) is given by

$$
w^{\varepsilon}>w-p\left(\frac{1}{\tau}\right) \text { and } \tau^{*}>\tau_{\min } .
$$

Note that if

$$
v^{\varepsilon}>w-p\left(\frac{1}{\tau}\right),
$$

then we will always be in Case 2, and therefore due to equation (3.30) there is a collision for any value $\tau_{\min }>0$ provided $\varepsilon$ is sufficiently small. Condition (3.34) shows the interplay of the "carelessness" of the drivers (characterized by $\varepsilon$ ) and the initial velocity and density of the surrounding cars. In [29] only the case $\varepsilon=0$ has been considered and this is related to completely "careless" drivers and the presented condition coincides with (3.34). Also, in this case the total mass is not conserved. In Eulerian coordinates a $\delta$-shock is observed which is not a solution in sense of Definition 3.1.

REMARK 3.5. In real-word scenarios, at the instant of a rear end collision the driver in front would ideally stop. However, this also depends on the dynamics of the car in the rear. Our model captures this fact as follows. Because the model does not allow cars to overtake each other, then when a collision occurs we have $\tau^{*} \longrightarrow 0$; hence in 


\section{LAGRANGIAN MACROSCOPIC MODELLING OF ROAD TRAFFIC COLLISIONS}

that situation, the $1^{\varepsilon}$-shock wave in Case 2 of Proposition 3.4, which connects the state $U^{\varepsilon}=\left(\tau^{\varepsilon}, w^{\varepsilon}\right)$ to the intermediate state $U^{*}=\left(\tau^{*}, w^{\varepsilon}\right)$ is such that $v^{*}=\min (0, w-$ $p(1 / \tau))$. Eventually the speed of the leading careless driver vanishes, and because $U^{*}$ is connected to $U=(\tau, w)$ through a contact discontinuity i.e. $v=v^{*}$, then so does the speed of the last driver within the AR regime. Furthermore, because the wave of the second family is a contact discontinuity then both cars involved in the collision remain parked.

\section{Numerical experiments}

This section is dedicated to some numerical simulations to illustrate traffic dynamics in the coupled model (3.28)-(3.29), using some sample initial traffic conditions. The AR model (3.28) is solved using a classical Godunov scheme where the Riemann solver is derived from analytical results presented above. Let us introduce some grid points in space $\left(X_{j}=j \Delta X, j \in \mathbb{Z}\right)$ and in time $\left(T_{n}=n \Delta T, n \in \mathbb{N}\right)$, with $(\Delta X, \Delta T) \longrightarrow(0,0)$ and $\frac{\Delta T}{\Delta X}=$ constant such that the CFL condition is satisfied. The space step $\Delta X$ can be viewed, at the microscopic level, as the average length of a car. Then, the Godunov discretization of the AR model in Lagrangian coordinates writes

$$
\begin{aligned}
& \tau\left(X_{i}, T_{n+1}\right)=\tau\left(X_{i}, T_{n}\right)+\frac{\Delta T}{\Delta X}\left[v\left(X_{i+1}, T_{n}\right)-v\left(X_{i}, T_{n}\right)\right], \\
& w\left(X_{i}, T_{n+1}\right)=w\left(X_{i}, T_{n}\right),
\end{aligned}
$$

where $v\left(X_{k}, T_{n}\right)=w\left(X_{k}, T_{n}\right)-p\left(\frac{1}{\tau\left(X_{k}, T_{n}\right)}\right)$.

Using the relationships $\partial_{T} x=\partial_{t} x=v$ and $T=t$ from (3.5), the location of the $i^{\text {th }}$ vehicle, in Eulerian coordinates, is given by

$$
x_{i}\left(t_{n+1}\right)=x_{i}\left(t_{n}\right)+\Delta t v\left(X_{i}, t_{n}\right) \text {. }
$$

Similarly, the Godunov discretization of the $\mathrm{AR}^{\varepsilon}$ model in Lagrangian coordinates (3.29) writes

$$
\begin{aligned}
& \tau^{\varepsilon}\left(X_{i}, T_{n+1}\right)=\tau^{\varepsilon}\left(X_{i}, T_{n}\right)+\frac{\Delta T}{\Delta X}\left[v^{\varepsilon}\left(X_{i+1}, T_{n}\right)-v^{\varepsilon}\left(X_{i}, T_{n}\right)\right] \\
& w^{\varepsilon}\left(X_{i}, T_{n+1}\right)=w^{\varepsilon}\left(X_{i}, T_{n}\right)
\end{aligned}
$$

where $v^{\varepsilon}\left(X_{k}, T_{n}\right)=w^{\varepsilon}\left(X_{k}, T_{n}\right)-\varepsilon p\left(\frac{1}{\tau^{\varepsilon}\left(X_{k}, T_{n}\right)}\right)$, with $\varepsilon \longrightarrow 0$.

Thanks to $(3.5)$, the location of the $i^{\text {th }}$ vehicle, in Eulerian coordinates, is given by

$$
x_{i}\left(t_{n+1}\right)=x_{i}\left(t_{n}\right)+\Delta t v^{\varepsilon}\left(X_{i}, t_{n}\right) .
$$

For the numerical simulations, we consider a road section with two platoons of vehicles, as depicted in figure 3.5. The first platoon consists of twenty vehicles (on the right part of the road section), for which the traffic dynamics are governed by the AR system (3.28), whereas the second platoon consists of five vehicles (on the left-end of the road section), for which the traffic regime is described by the $\mathrm{AR}^{\varepsilon}$ model for a parameter $\varepsilon=O\left((\Delta X)^{4}\right)$. The choice of $\varepsilon$ implies that numerically we are close to the regime of the PGD model (2.9)-(2.10). Initially the vehicles' positions are randomly prescribed in both regimes. For both $\mathrm{AR}$ and $\mathrm{AR}^{\varepsilon}$ models, we consider normalized values for the variable $\tau$, i.e. $\tau_{\min }=1 / \rho_{\max }=1$, where $\rho_{\max }$ is the maximal 
density i.e. when vehicles are parked bumper-to-bumper, and we set $p(\rho)=\rho^{\gamma}$, with $\gamma=1$. Therefore, in the simulations, a collision is characterized by the occurrence of a $\delta$-solution (i.e., $\tau<\tau_{\min }$ ) in the $\mathrm{AR}^{\varepsilon}$ model. This is equivalent to a situation where the leader in the platoon governed by the $\mathrm{AR}^{\varepsilon}$ model collides with the last vehicle in the platoon governed by the $\mathrm{AR}$ model, i.e. the distance between the rear ends of these two vehicles is less than the average length of a vehicle, $\Delta X$.

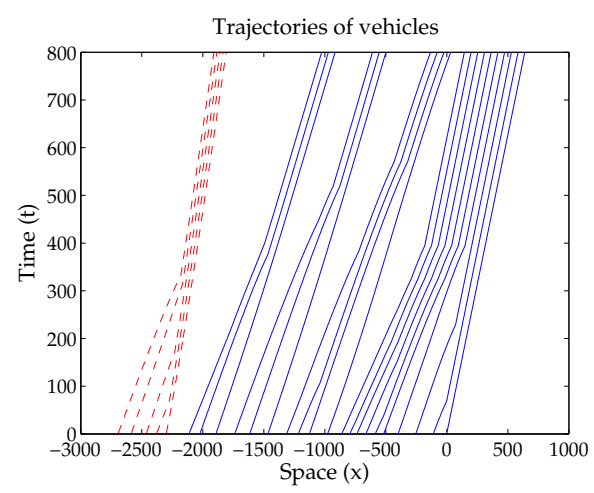

(a)

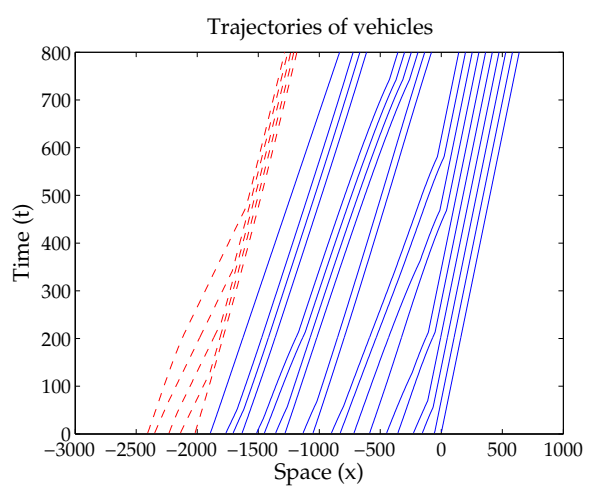

(b)

FIG. 4.1. (a) The initial setting in the $A R^{\varepsilon}$ regime is $w^{\varepsilon}\left(X_{j}, 0\right)=0.5, \forall j=1, \ldots 5$, with $w^{\varepsilon}<$ $w\left(X_{20}, t\right)-p\left(\tau\left(X_{20}, t\right)\right), \forall t$. (b) The initial setting in the $A R^{\varepsilon}$ regime is $w^{\varepsilon}\left(X_{j}, 0\right)=0.8, \forall j=1, \ldots 5$, with $w^{\varepsilon}<w\left(X_{20}, t\right)-p\left(\tau\left(X_{20}, t\right)\right), \forall t$.

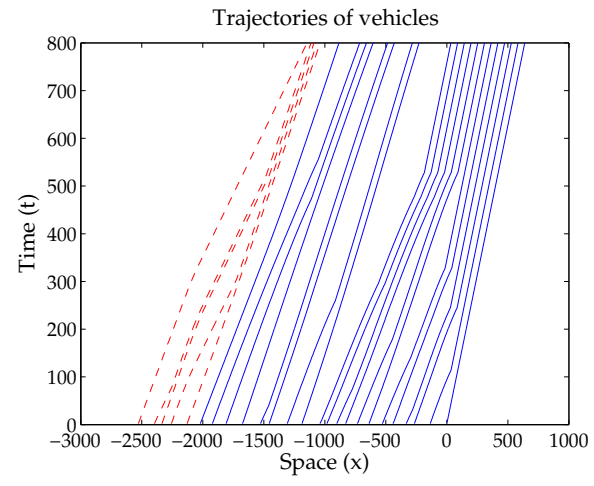

(a)

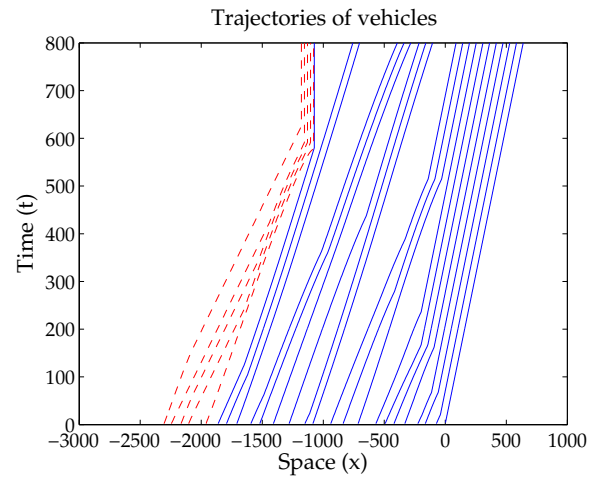

(b)

FIG. 4.2. (a) The initial setting in the $A R^{\varepsilon}$ regime is $w^{\varepsilon}\left(X_{j}, 0\right)=1, \forall j=1, \ldots 5$, with $w^{\varepsilon}=$ $w\left(X_{20}, t\right)-p\left(\tau\left(X_{20}, t\right)\right), \forall t$. (b) The initial setting in the $A R^{\varepsilon}$ regime is $w^{\varepsilon}\left(X_{j}, 0\right)=1, \forall j=1, \ldots 5$, with $w^{\varepsilon}>w\left(X_{20}, t\right)-p\left(\tau\left(X_{20}, t\right)\right)$.

The results of the numerical simulations are presented in terms of trajectories of vehicles in space and time (in Eulerian coordinates) in figures 4.1, 4.2, and 4.3. In all the figures, the solid lines correspond to the trajectories of vehicles within the AR regime whereas the dashed lines correspond to trajectories of vehicles in the $\mathrm{AR}^{\varepsilon}$ regime. Throughout the simulations, the following setting has been adopted for the AR regime: we set $w\left(X_{i}, 0\right)=1, \forall i=1, \ldots, 20$, i.e. the maximal speed allowed in 


\section{LAGRANGIAN MACROSCOPIC MODELLING OF ROAD TRAFFIC COLLISIONS}

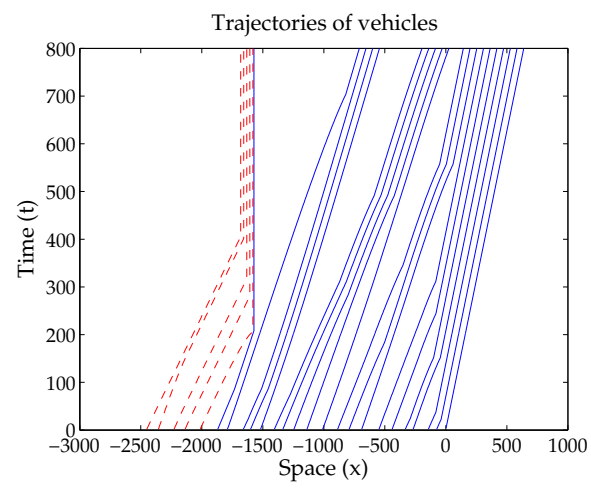

(a)

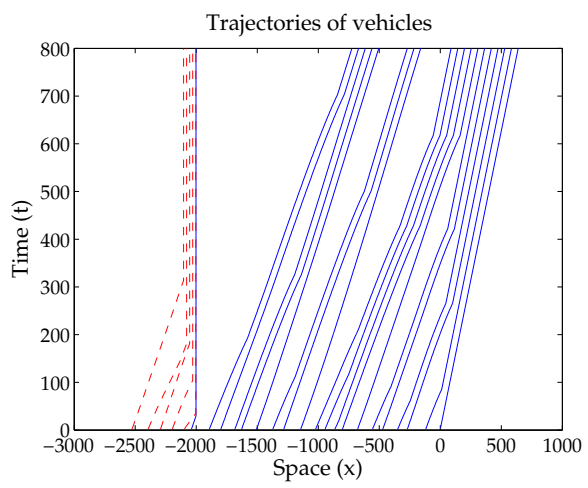

(b)

FIG. 4.3. (a) The initial setting in the $A R^{\varepsilon}$ regime is $w^{\varepsilon}\left(X_{j}, 0\right)=1.5, \forall j=1, \ldots 5$, i.e. $w^{\varepsilon}>$ $w\left(X_{20}, t\right)-p\left(\tau\left(X_{20}, t\right)\right), \forall t$. (b) The initial setting in the $A R^{\varepsilon}$ regime is $w^{\varepsilon}\left(X_{j}, 0\right)=1.8, \forall j=1, \ldots 5$, i.e. $w^{\varepsilon}>w\left(X_{20}, t\right)-p\left(\tau\left(X_{20}, t\right)\right), \forall t$.

the regime is 1 , and the initial positions of vehicles are prescribed randomly such that the initial velocity for each vehicle, deduced uniquely using the relationship $v=w-p(\rho)$ with $\rho=\Delta X /\left(x_{i+1}-x_{i}\right)$, is less than or equal to 1 . For the leading vehicle in the AR regime, we consider a constant speed of 0.8 . Then, we illustrate the scenarios summarized in Proposition 3.4 by varying the settings in the $\mathrm{AR}^{\varepsilon}$ regime while $\varepsilon=10^{-5}$.

The results portrayed in figure 4.1 (a) and (b) are obtained using the following settings in the $\mathrm{AR}^{\varepsilon}$ regime. In figure 4.1 (a), we set $w^{\varepsilon}\left(X_{i}, 0\right)=0.5 \forall i$, i.e. the maximal speed allowed in the regime is 0.5 , and the initial positions of vehicles are prescribed randomly such that the initial velocity for each vehicle, deduced uniquely using the relation $v=w-p(\rho)$ with $\rho=\Delta X /\left(x_{i+1}-x_{i}\right)$, is less than or equal to 0.5 . In figure $4.1(\mathrm{~b})$, we set $w^{\varepsilon}\left(X_{i}, 0\right)=0.8 \forall i$, i.e. the maximal speed allowed in the regime is 0.8 , and the initial positions of vehicles are prescribed randomly such that the initial velocity for each vehicle, deduced uniquely using the relationship $v=w-p(\rho)$ with $\rho=\Delta X /\left(x_{i+1}-x_{i}\right)$, is less than or equal to 0.8. The results in figure 4.1 (a) and (b) correspond to the case $w^{\varepsilon}<w-p(1 / \tau)$ (Case 2 in Proposition 3.4), and as shown in the graphs, the solution consists of two contact discontinuities (with speeds $w^{\varepsilon}$ and $w-p(1 / \tau)$ respectively) separated by a vacuum state.

In figure 4.2 (a) and (b), the following settings were considered for the $\mathrm{AR}^{\varepsilon}$ regime: $w^{\varepsilon}\left(X_{i}, 0\right)=1 \forall i$, i.e. the maximal speed allowed in the regime is 1 , and the initial positions of vehicles are prescribed randomly such that the initial velocity for each vehicle, deduced uniquely using the relationship $v=w-p(\rho)$ with $\rho=\Delta X /\left(x_{i+1}-x_{i}\right)$, is less than or equal to 1. Figure 4.2 (a) corresponds to the situation where $w^{\varepsilon}=$ $w-p(1 / \tau)$ (Case 1 in Proposition 3.4), and as shown by the graph, the solution consists of a contact discontinuity traveling with speed $w-p(1 / \tau)$. Figure $4.2(\mathrm{~b})$ corresponds to the high-risk situation where $w^{\varepsilon}>w-p(1 / \tau)$ (Case 3 in Proposition 3.4 ), and as illustrated in the graph a collision occurs at $t=480$ between the last vehicle in the $\mathrm{AR}$ regime and the leading vehicle in the $\mathrm{AR}^{\varepsilon}$ regime.

The results depicted in figure 4.3 (a) and (b) are obtained using the following settings in the $\mathrm{AR}^{\varepsilon}$ regime. In figure 4.3 (a) we set $w^{\varepsilon}\left(X_{i}, 0\right)=1.5 \forall i$, i.e. the maximal speed allowed in the regime is 1.5 , and the initial positions of vehicles are prescribed 
randomly such that the initial velocity for each vehicle, deduced uniquely using the relation $v=w-p(\rho)$ with $\rho=\Delta X /\left(x_{i+1}-x_{i}\right)$, is less than or equal to 1.5. In figure 4.3 (b) we set $w^{\varepsilon}\left(X_{i}, 0\right)=1.8 \forall i$, i.e. the maximal speed allowed in the regime is 1.8 , and the initial positions of vehicles are prescribed randomly such that the initial velocity for each vehicle, deduced uniquely using the relationship $v=w-p(\rho)$ with $\rho=\Delta X /\left(x_{i+1}-x_{i}\right)$, is less than or equal to 1.8. In both cases we have $w^{\varepsilon}>w \geq$ $w-p(1 / \tau)$ (Case 3 in Proposition 3.4), and therefore both cases correspond to the high-risk situation where a collision is likely to occur. As shown in the graph, in figure 4.3 (a) a collision between the last vehicle in the AR regime and the leading vehicle in the $\mathrm{AR}^{\varepsilon}$ regime occurs at $t=270$, whereas in figure 4.3 (b) the collision occurs at $t=60$.

Acknowledgment. This work has been supported by DFG SPP1253 HE5386/71, DAAD D/08/11076, 50727872, and HE5386/8-1. The authors are grateful to the anonymous referees whose valuable remarks enable to improve to the paper.

\section{REFERENCES}

[1] A. Aw, A. Klar, T. Materne, and M. Rascle, Derivation of continuum traffic flow models from microscopic follow-the-leader models, SIAM J. Appl. Math., 63, 259-278, 2002.

[2] A. Aw and M. Rascle, Resurrection of "second order" models of traffic flow, SIAM J. Appl. Math., 60, 916-938, 2000.

[3] M.K. Banda, M. Herty, and A. Klar, Coupling conditions for gas networks governed by the isothermal Euler equations, Netw. Heterog. Media, 1, 295-314, 2006.

[4] F. Berthelin, P. Degond, M. Delitala, and M. Rascle, A model for the formation and evolution of traffic jams, Arch. Ration. Mech. Anal., 187, 185-220, 2008.

[5] F. Berthelin, P. Degond, V. Le Blanc, S. Moutari, M. Rascle, and J. Royer, A traffic-flow model with constraints for the modeling of traffic jams, Math. Models Meth. Appl. Sci., 18, 1269-1298, 2008.

[6] F. Bouchut, On zero pressure gas dynamics, in Advances in Kinetic Theory and Computing, of Ser. Adv. Math. Appl. Sci., World Sci. Publ., River Edge, NJ, 22, 171-190, 1994.

[7] Y. Brenier and E. Grenier, Sticky particles and scalar conservation laws, SIAM J. Numer. Anal., 35, 2317-2328, 1998.

[8] B.S.C. Lank and A. Smmermann, Bewertungsverfahren für Verkehrs- und Verbindungsqualitten von Hauptverkehrsstraen, Berichte der Bundesanstalt für Straenwesen, Heft, 183, 2009.

[9] R. Chandler, R. Herman, and E.W. Montroll, Traffic dynamics: Studies in car following, Oper. Res., 6, 165-184, 1958.

[10] G.Q. Chen and H. Liu, Formation of $\delta$-shocks and vacuum states in the vanishing pressure limit of solutions to the Euler equations for isentropic fluids, SIAM J. Math. Anal., 34, 925-938, 2003.

[11] A. Chertock and A. Kurganov, Computing multivalued solutions of pressureless gas dynamics by deterministic particle methods, Commun. Comput. Phys., 5, 565-581, 2009.

[12] A. Chertock, A. Kurganov, and Y. Rykov, A new sticky particle method for pressureless gas dynamics, SIAM J. Numer. Anal., 45, 2408-2441, 2007.

[13] G.M. Coclite, M. Garavello, and B. Piccoli, Traffic flow on a road network, SIAM J. Math. Anal., 36, 1862-1886, 2005.

[14] R.M. Colombo and M. Garavello, On the Cauchy problem for the p-system at a junction, SIAM J. Math. Anal., 39, 1456-1471, 2008.

[15] R.M. Colombo, G. Guerra, M. Herty, and V. Schleper, Optimal control in networks of pipes and canals, SIAM J. Control Optim., 48, 2032-2050, 2009.

[16] C.F. Daganzo, Requiem for second order fluid approximations of traffic flow, Trans. Res. B, 29, 277-287, 1995.

[17] C.F. Daganzo, A behavioral theory of multi-lane traffic flow part i: Long homogeneous freeway sections, Trans. Res. B, 36, 131-158, 2002.

[18] J. de Halleux, C. Prieur, J.M. Coron, B. d'Andréa Novel, and G. Bastin, Boundary feedback control in networks of open channels, Automatica J. IFAC, 39, 1365-1376, 2003.

[19] P. Degond, S. Göttlich, M. Herty, and A. Klar, A network model for supply chains with multiple policies, Multiscale Model. Simul., 6, 820-837, 2007. 


\section{LAGRANGIAN MACROSCOPIC MODELLING OF ROAD TRAFFIC COLLISIONS}

[20] H.T. Fritzsche, A model for traffic simulation, Traffic Engin. Control, 35, 317-321, 1994.

[21] M. Garavello and B. Piccoli, Traffic flow on a road network using the Aw-Rascle model, Commun. Part. Diff. Eqs., 31, 243-275, 2006.

[22] M. Garavello and B. Piccoli, Traffic Flow on Networks, AIMS Series on Applied Mathematics, American Institute of Mathematical Sciences (AIMS), Springfield, MO, 1, 2006.

[23] P.G. Gipps, A behavioural car-following model for computer simulation, Transport. Res. B, 15, 105-111, 1981.

[24] M. Gugat, G. Leugering, K. Schittkowski, and E.J. P.G. Schmidt, Modelling, stabilization, and control of flow in networks of open channels, in Online Optimization of Large Scale Systems, Springer, Berlin, 251-270, 2001,

[25] M. Herty and R. Illner, Analytical and numerical investigations of refined macroscopic traffic flow models, Kin. Rel. Mod., 3, 311-333, 2010.

[26] M. Herty, C. Kirchner, S. Moutari, and M. Rascle, Multicommodity flows on road networks, Commun. Math. Sci., 6, 171-187, 2008.

[27] M. Herty and A. Klar, Modeling, simulation, and optimization of traffic flow networks, SIAM J. Sci. Comput., 25, 1066-1087, 2003.

[28] M. Herty and A. Klar, Simplified dynamics and optimization of large scale traffic networks, Math. Models Meth. Appl. Sci., 14, 579-601, 2004.

[29] M. Herty, A. Klein, S. Moutari, V. Schleper, and G. Steinaur, Modeling road traffic accidents using macroscopic second-order models of traffic flow, IMA J. Appl. Math., 78(5), 10871108, 2013.

[30] M. Herty and M. Rascle, Coupling conditions for a class of second-order models for traffic flow, SIAM J. Math. Anal., 38, 595-616 (electronic), 2006.

[31] M. Herty and V. Schleper, Traffic flow with unobservant drivers, ZAMM J. Appl. Math. Mech., 91, 763-776, 2011.

[32] H. Holden and N. H. Risebro, A mathematical model of traffic flow on a network of unidirectional roads, SIAM J. Math. Anal., 26, 999-1017, 1995.

[33] J. Hu, Two-dimensional Riemann problem for pressureless gas dynamics equations with functional solutions, Quart. Appl. Math., 58, 251-264, 2000.

[34] P.D. Lax, Hyperbolic Systems of Conservation Laws and the Mathematical Theory of Shock Waves, CBMS-NSF Regional Conference Series in Applied Mathematics, New York, 1973.

[35] P. LeFloch and T.P. Liu, Existence theory for nonlinear hyperbolic systems in nonconservative form, Forum Math., 5, 261-280, 1993.

[36] M.J. Lighthill and J.B. Whitham, On kinematic waves, Proc. Royal Soc. Edinburgh, A229, 281-345, 1955.

[37] H. Payne, FREFLO: A macroscopic simulation model for freeway traffic, Transport. Res. Record, 722, 68-77, 1979.

[38] P. Richards, Shock waves on the highway, Oper. Res., 4, 42-51, 1956.

[39] E.P. Todosiev, The Action Point Model of the Vehicle-Driver System, Technical Report 202A-3, Ohio State University, 1963.

[40] D. Wagner, Equivalence of the Euler and Lagrangian equations of gas dynamics for weak solutions, J. Diff. Eqs., 68, 118-136, 1987.

[41] R. Wiedemann, Simulation des straenverkehrsflusses, Schriftenreihe des Instituts für Verkehrswesen der Universitt Karlsruhe, 1974.

[42] H.M. Zhang, A non-equilibrium traffic model devoid of gas-like behaviour, Transport. Res. B, $36,275-298,2002$. 